\title{
Interferometric gravitational wave detectors vibrational isolation
}

\section{Riccardo DeSalvo}

Riccardo DeSalvo, "Interferometric gravitational wave detectors vibrational isolation," Proc. SPIE 4093, Current Developments in Lens Design and Optical Systems Engineering, (24 October 2000); doi: 10.1117/12.405247

Event: International Symposium on Optical Science and Technology, 2000, San Diego, CA, United States 


\title{
Interferometric Gravitational Wave Detector s Vibrational Isolation
}

\author{
Riccardo DeSalvo \\ LIGO Project, MS 18-34 \\ California Institute of Technology \\ Pasadena, CA 91125
}

\begin{abstract}
Interferometric Gravitational Wave Detectors, coming online in late 2000, look for small space strains, leading to apparent motions of test masses of $10-19 \mathrm{~m}$ or less; isolation from other forces is crucial. They require a formidable vibration isolation level in a frequency range between few $\mathrm{Hz}$ and few $\mathrm{KHz}$. The off-band residual motion must be kept below 10-12 $\mathrm{m}$ not to saturate the phase sensors. These exceptional requirements are met, in all degrees of freedom, with a chain of active and passive filters. The key isolation mechanism is the use of mechanical oscillators above their resonant frequencies, pendula horizontally, springs vertically. Very high Quality pendular suspensions are needed at the mirror level to limit the "thermal" noise from fluctuations in the dissipation mechanisms. Off-band electromagnetic actuators on or near the mirror keep its residual motion within the required limits. These final mirror suspension stages only provide the final few orders of magnitude of attenuation in the longitudinal direction. To provide the bulk of the attenuation, virtually all in the vertical direction, they are suspended from Seismic Noise Attenuation Systems. Attenuation filters, either active or passive, are chained, each providing 2 or 3 orders of magnitude of attenuation. Passive attenuation is obtained with springs and pendula. The vertical is the toughest direction to deal with because the oscillators also fight against gravity. The vertical attenuation requirements, although orthogonal to the beam direction, are only slightly less stringent than the vertical ones due to crosscouplings (Earth curvature is the source of one of them). High internal damping springs organised in hierarchical stacks are used in most early designs. More advanced designs increasingly rely on chains of filters equipped with high quality cantilever springs driven to low resonant frequencies by different mechanisms. The Quality Factors of each resonance are actively and/or passively spoiled at the chain suspension point. In the latest designs, Ultra Low Frequency Oscillators filter out the "microseismic" and other low frequency perturbations. This paper addresses one approach to achieving the required seismic isolation level.
\end{abstract}

Keywords: Gravitational waves, seismic attenuation, passive attenuation

\section{INTRODUCTION}

Gravitational waves emitted by compact body (neutron stars and black holes) collisions are expected to radiate across the universe and slightly perturb any mass that they traverse. The differential movement imparted by gravitational waves to two test masses is proportional to their separation; consequently a gravitational wave detector must be built with widely separated masses. Separations of the order of kilometers are considered on Earth based detectors (LIGO, Virgo, TAMA, GEO, ACIGA $^{12}$ ), million of kilometers for space based interferometers $\left(\right.$ LISA $\left.^{3}\right)$. A typical gravitational wave event is expected to move masses a few kilometers apart by $10^{-18} \mathrm{~m}$ or less. It has been shown that it is possible, by means of laser interferometric techniques in Ultra High Vacuum, to measure such small distance variations. The $10^{-18} \mathrm{~m}$ signal is an outrageously small number that has to be compared with, and isolated from, the continuous, up to $10^{-6} \mathrm{~m}$, seismic random motion of Earth s crust. Seismic Isolation (SI) is therefore one of the key components of a Gravitational Wave Interferometric Detector (GWID). Gravitational waves are, in principle, emitted at all orbital frequencies, with an upper limit given by the dimension of the compact bodies of interest (kilometers) divided by the speed of light, which is reached at the final stage of coalescence. On Earth, at frequencies lower than a few $\mathrm{Hz}$, the gravitational wave signal is swamped by the fluctuations of the gravitational pull of the Earth s crust and there is presently no way to recover the signal. Earth based GWIDs can therefore be designed to work only above a few Hz. The SI of a GWID is required to provide a staggering $10^{-12}$, or better, attenuation factor within a frequency range from a few $\mathrm{Hz}$ to a few $\mathrm{kHz}$. There are additional requirements. In principle the test masses could swing freely at all frequencies below the frequency region of interest. At these frequencies all suspended systems tend to oscillate at their own pendulum resonant frequencies with amplitudes proportional to the excitation amplitudes and to their mechanical quality factors. The interferometric displacement sensing with $<10^{-18} \mathrm{~m}$ resolution has a dynamic range of the order of a million and may loose track of the position if the mirrors move, even at low frequency, more than $10^{-12} \mathrm{~m}$ from the exact dark fringe position. The SI and mirror suspension system must therefore 
provide positioning control of the mirror with an absolute precision much better than $10^{-12} \mathrm{~m}$ at low to DC frequencies ${ }^{4}$. Not surprisingly, actuators with less than $10^{-18} \mathrm{~m}$ noise and resolution have very limited dynamic range. The $10^{-12} \mathrm{~m}$ requirement then sets a limit on how much energy can be allowed to be stored in the mechanical resonances of the SI and suspension system.

All of these requirements can be satisfied with a series of layered defenses, the seismic attenuators, and with proper control authority distribution for positioning.

\section{SEISMIC ATTENUATION SCHEME}

The most promising solution for the seismic attenuation task is represented by the superattenuator chains first introduced by Virgo and further developed at LIGO for LIGO II and TAMA. The design and results presented here have been achieved in the TAMA and LIGO prototypes. A typical Seismic Attenuation and Mirror Suspension System (Virgo, TAMA, LIGO II) consists of three parts:

- A very low frequency pre-isolator to absorb the effects of the micro-seismic peak and provide positioning.

- A chain of passive mechanical filters to stop the bulk of the seismic noise within the frequency band of interest.

- A multiple pendulum built from quartz or other low dissipation materials to shield the mirror from thermal noise and provide the mirror controls.

The attenuation is obtained by the fact that an oscillator, excited at frequencies higher than its own resonant frequency, will transmit that excitation with an attenuation factor falling in accordance with to the $1 / \mathbf{f}^{2}$ law. Chained oscillators simply multiply their attenuation factors. A seismic attenuation chain is composed of a sufficient number of low frequency oscillators (pendulums horizontally, springs vertically). A well-designed attenuation system can generate the necessary inband attenuation completely passively. Mechanical oscillators saturate their $1 / \mathrm{f}^{2}$ behavior at high frequencies because of the short circuiting effect generated by the moment of inertia of the mass distributed across their structure. These limitations, illustrated in figure 1 , will be discussed individually in the following sections.

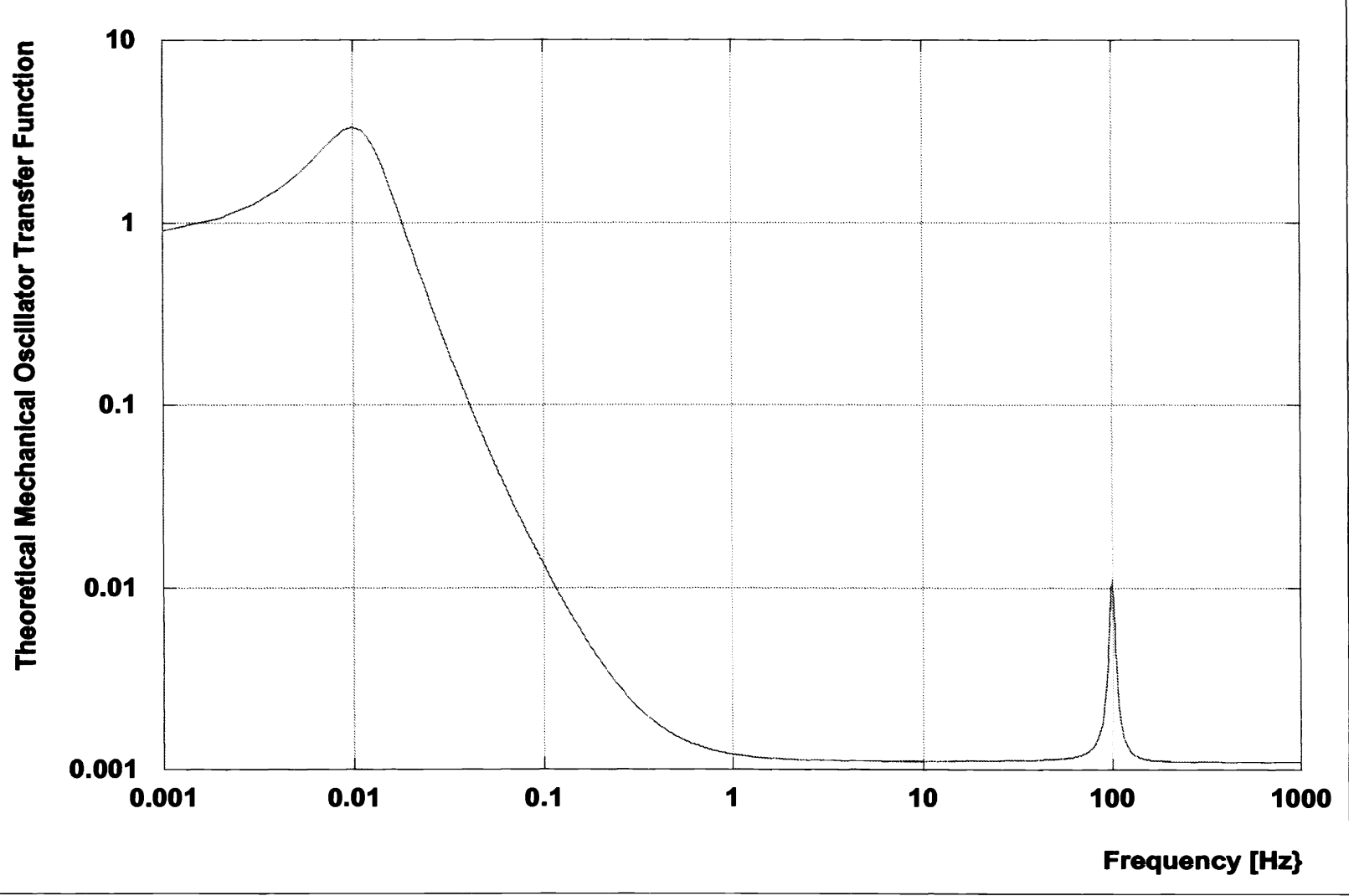

Figure 1: Theoretical attenuation performance of a single passive attenuation filter. The peak at $10 \mathrm{mHz}$ is the main resonance of the oscillator; it is followed by the characteristic $1 / \mathrm{f}^{2}$ attenuation function, which is interrupted at $1 \mathrm{~Hz}$ by saturation due to the distributed mass in the oscillator mechanics. The peak at $100 \mathrm{~Hz}$ is given by the internal resonances of the mechanics The frequencies and attenuation factors are arbitrarily chosen but close to what is actually feasible. This topology is typical for all passive attenuation filters. 
The fundamental resonances of a SI oscillator chain are typically located between 0.1 and $3 \mathrm{~Hz}$. If excited, these resonances would gently swing the mirror well beyond the dynamic range of the position sensors. It is therefore necessary to shield these resonances from seismic excitation and drain any energy stored in them. This job is performed by the pre-isolator operating mainly below the frequency region of interest $(10 \mathrm{mHz}$ to $10 \mathrm{~Hz})$. Although it may also provide substantial in band attenuation, its main function is to stabilize and position the rest of the passive attenuation chain to within a small fraction of a micron. The attenuation is in large part passive while the extraction of the energy from the resonances is achieved via voice coil actuators in feedback over the signal of sensitive accelerometers.

On the high end of the frequency range, the oscillator $s$ attenuation factor is saturated by distributed mass effects and can be spoiled if uncontrolled internal resonances are present. The attenuation saturation due to the distributed mass (batting center problem) can be minimized by carefully shaping the moving components of the oscillator ${ }^{5}-{ }^{6}$. The internal resonances must be controlled by careful engineering.

Finally a SI must not generate more mechanical noise that it filters out: creep and creak are the main sources of noise. Creep events may jerk the attenuation chain by as much as $10^{-12} \mathrm{~m}$. A well designed SI must be creak free and must control the creep level within the required sensitivity level. The materials must have high quality factors not to introduce thermal noise to the system.

In the following the different components of a SI will be presented in some detail. An overall view of the TAMA Seismic Attenuation Chain is shown in figure 2.
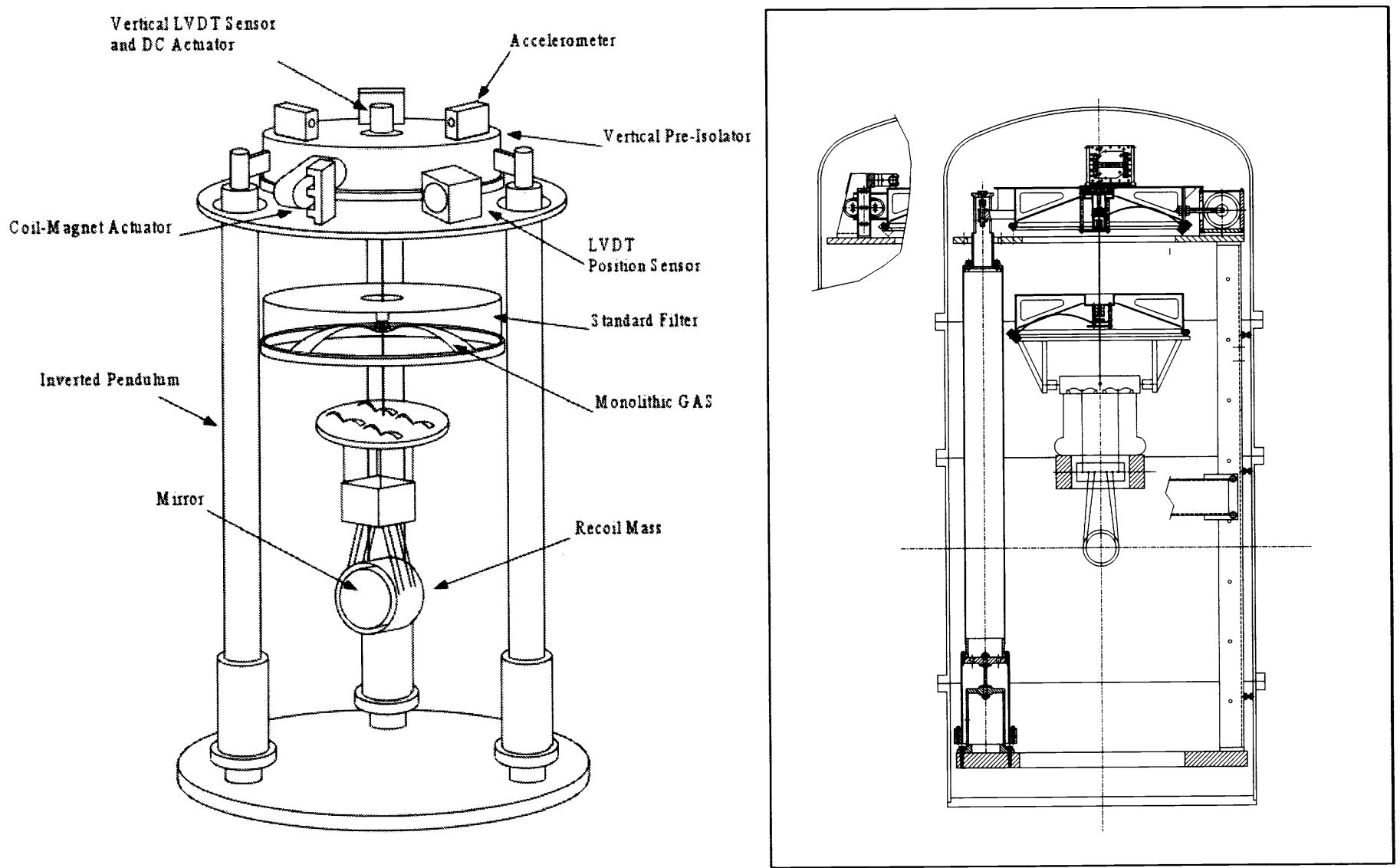

Figure 2: TAMA seismic attenuation chain. The pre-attenuation stage is composed by an inverted pendulum and a first filter provided with accelerometers, LVDTs and actuators to provide inertial damping of the chain $s$ body modes. The seismic attenuation in the TAMA configuration case is provided by a single MGASF filter followed by a triple pendulum mirror control system. 


\section{THE PRE-ISOLATOR}

The pre-isolator is made by an Inverted Pendulum (IP) supporting a vertical oscillation spring. The IP is made of three rigid legs mounted on stiff flex joints that provide the oscillator restoring force. The three legs are joined by a platform at the top. This platform is free to move in the horizontal plane in the two $\mathrm{x}$ and $\mathrm{y}$ degrees of freedom as well as in torsion around the vertical axis. The platform body contains a filter (see below) for the vertical degree of freedom. In order to be effective against the $150 \mathrm{mHz}$ micro-seismic peak, the IP is tuned at the lowest possible frequency, 10 to $30 \mathrm{mHz}$. This is achievable in IPs by tuning the load with the addition or removal of small ballast masses. One additional advantage of working at low frequencies is that, even with low loss materials, very low effective quality factors can be achieved. In the IP the attenuation saturation due to the legs distributed mass is particularly severe but it is also easily soluble with suitable counterweights below the flex joint. As proven with simulations and measurements by Virgo and AIGO, careful tuning of these counterweights allows the saturation plateau to be pushed down essentially with the tuning precision of the counterweight. In the TAMA and LIGO design, the IP performance should stay close to its theoretical $1 / \mathbf{f}^{2}$ behavior over all the attenuation chain resonances frequency range $(100 \mathrm{mHz}$ to $5 \mathrm{~Hz})$.

Despite the pre-isolator strong attenuation, the chain resonances would still be excited well above any acceptable level. The IP then is equipped with a set of three horizontal, low-frequency, high-sensitivity accelerometers controlling three low noise actuators to drain the residual seismic energy deposited in these resonances. Accelerometers and actuators are arranged in a triangular configuration to control also the yaw d.o.f. A computerized Multiple In Multiple Out control system transforms the signals and generates the damping forces down to the lowest frequencies $(\sim \mathrm{mHz})$ in each individual degree of freedom. The accelerometer sensitivity $\left(<10^{-10} \mathrm{~m}\right)$ can be used to generate the mirror positioning stability. A mirror stability of a few tens of $\mathrm{nm}$ is foreseen from the simulations, less than the required sub-micron off-band residual motion.

Finally, since the accelerometers do not sense position, at frequencies below $1 \mathrm{mHz}$ positioning of the IP is controlled by a mix of signals from auxiliary LVDT position sensors and the interferometer error signal itself acting through the MIMO actuator system.

The vertical degree of freedom is independently but similarly treated.

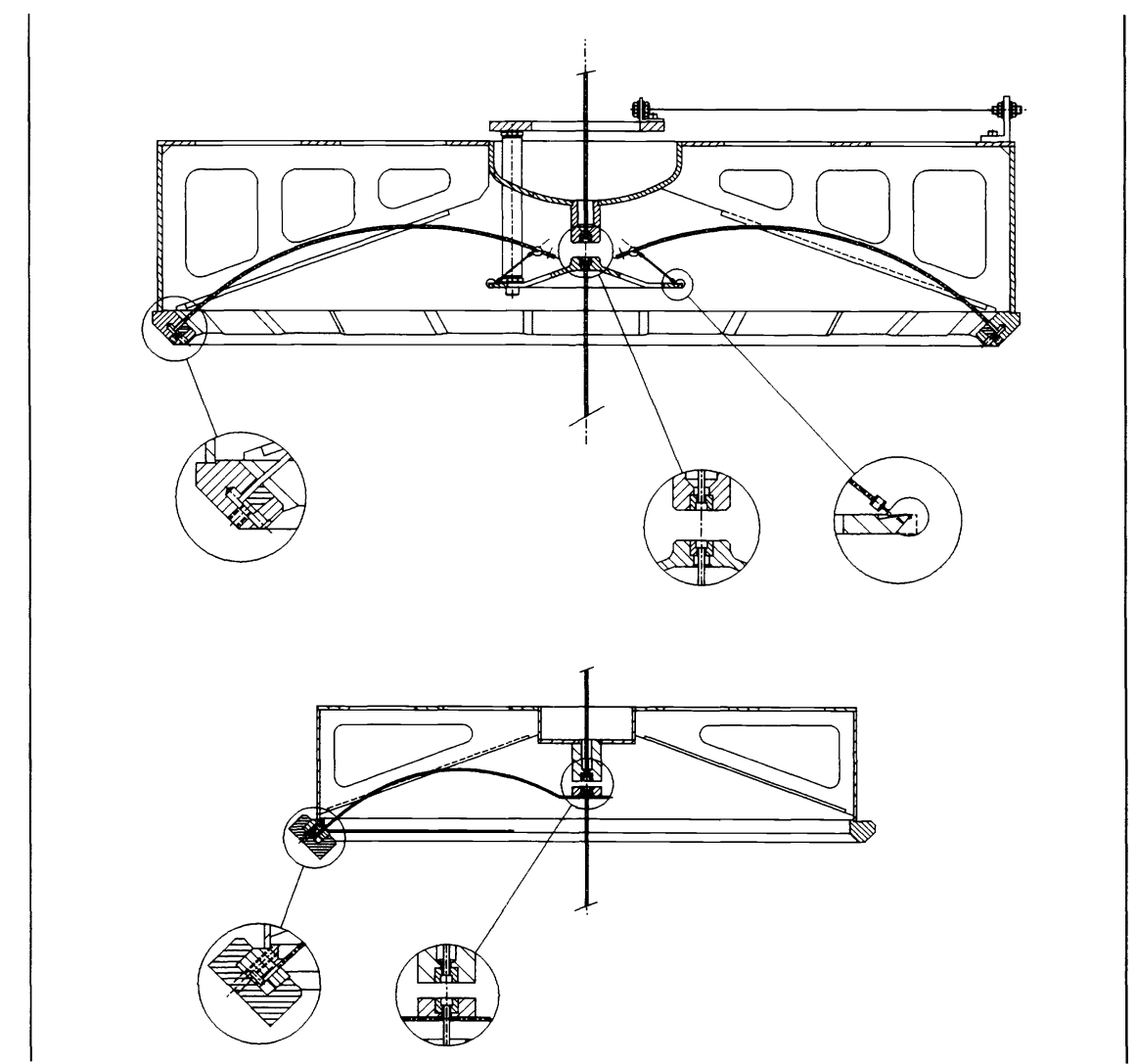

Figure 3: Section of a GASF (above) and of a MGASF (below). The antispring component responsible for the resonant frequency reduction is obtained by radial compression of the blades. In both designs any joint between separate parts is made through contact surfaces that are perpendicular to the stress direction (see enlargements) to avoid creak noise. 


\section{THE PASSIVE ISOLATOR FILTERS}

The bulk of the seismic attenuation is obtained using a chain of mechanical oscillators. In the horizontal direction this is trivially obtained by means of pendula. The suspension wire having negligible mass, a pendulum has an almost perfect $1 / \mathrm{f}^{2}$ attenuation up to the wire violin mode frequencies. With reasonable pendulum lengths (tens of centimeters) suitable attenuation factors can easily be achieved in the frequency band of interest. The difficult part in a good seismic isolator is to achieve good vertical attenuation. The vertical noise is, in theory, orthogonal to the sensitivity of the interferometer. Somewhere between a percent and a per-thousand of the vertical motion is transferred to the horizontal direction at each attenuation stage by mechanical imperfections, misalignments and, ultimately, by the non-parallelism of verticality in locations kilometers apart. The vertical attenuation then becomes practically as important as the others. Ideally a vertical oscillator can be obtained replacing the suspension wire with a massless spring working against the gravitational pull. To achieve sub-Hz vertical frequencies within the required space constraints, with acceptable stress levels and without coupling extension with rotation the pendulum masses in the attenuation chain are replaced by vertical attenuation filters. The filters are essentially compact springs, made of a crown of concentric cantilever springs ${ }^{78}$. The vertical oscillation frequency is achieved by means of various techniques. The novelty introduced at LIGO is the Geometric Anti Spring Filter ${ }^{9}$ (GASF) and, lately, Monolithic GASF (MGASF) shown in figure 3. In both schemes, the cantilever blades are radially compressed against the central point. At the filter working point the radial forces are in equilibrium. The frustrated radial stress tends to release itself by pushing the system up or down thus generating a negative spring potential. The amount of the negative spring constant thus produced can be tuned by changing the radial compression and the complexive vertical spring constant can be effectively nulled. Arbitrarily low resonant frequencies can be achieved at arbitrarily low material stress levels.

These low frequency springs are particularly stable because both the spring and the anti-spring constants derive from the same material. Their thermal variations are matched thus maintaining a fixed working frequency at the working point. The working point obviously changes with temperature because the real spring constant, producing the lift. This effect can be cured with a little bi-metal strip compensator.

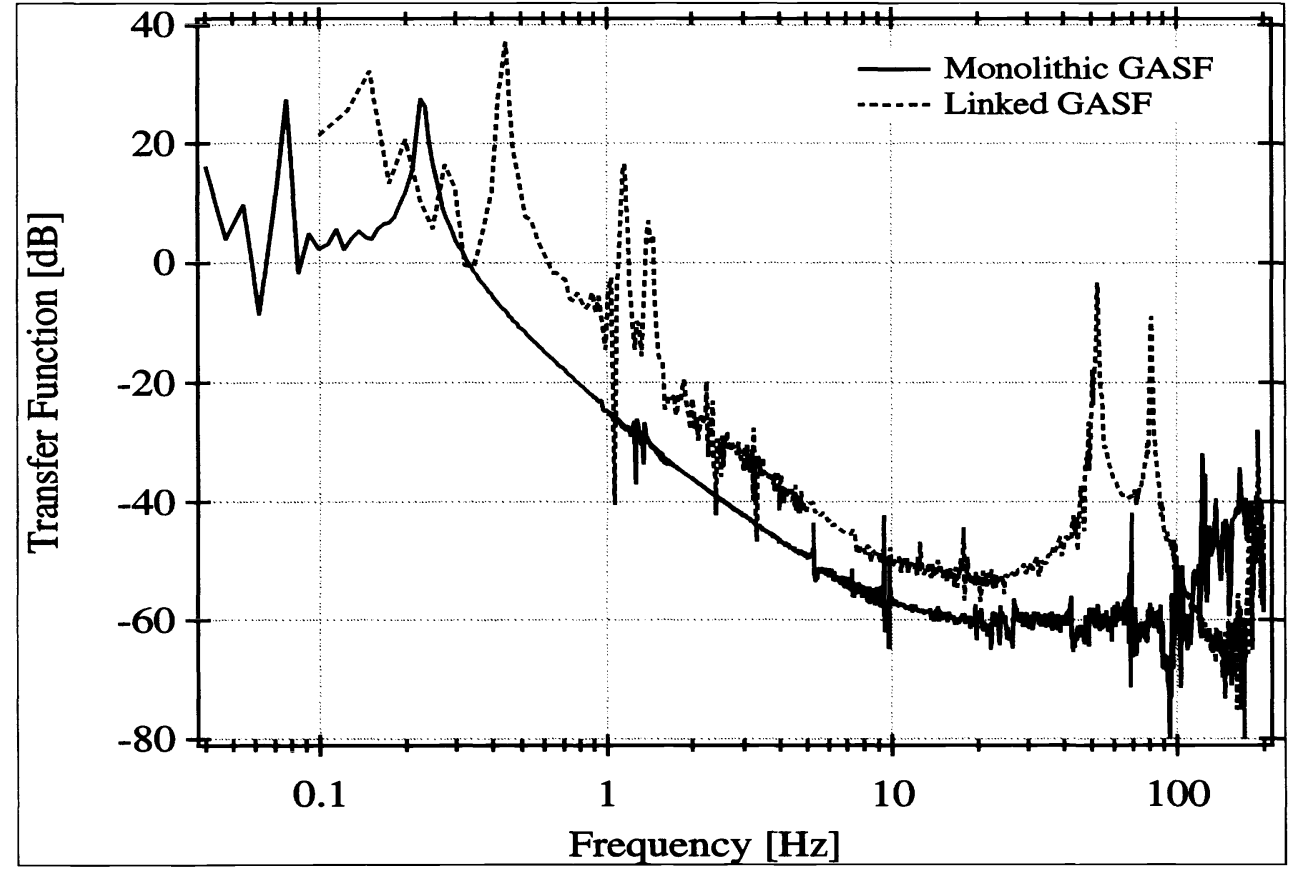

Figure 4: Attenuation transfer function of a GASF and a MGASF. The main vertical resonant frequency can be changed at will in both filters. The MGASF present a better attenuation plateau at medium frequencies and is effectively immune to the internal resonance visible at 50 and $80 \mathrm{~Hz}$ in the GASF transfer function. The peaks between 1 and $2 \mathrm{~Hz}$ and below $20 \mathrm{mHz}$ are payload tilt and rotational modes. At frequencies higher than $100 \mathrm{~Hz}$ acoustic transmission dominates the measurement. 

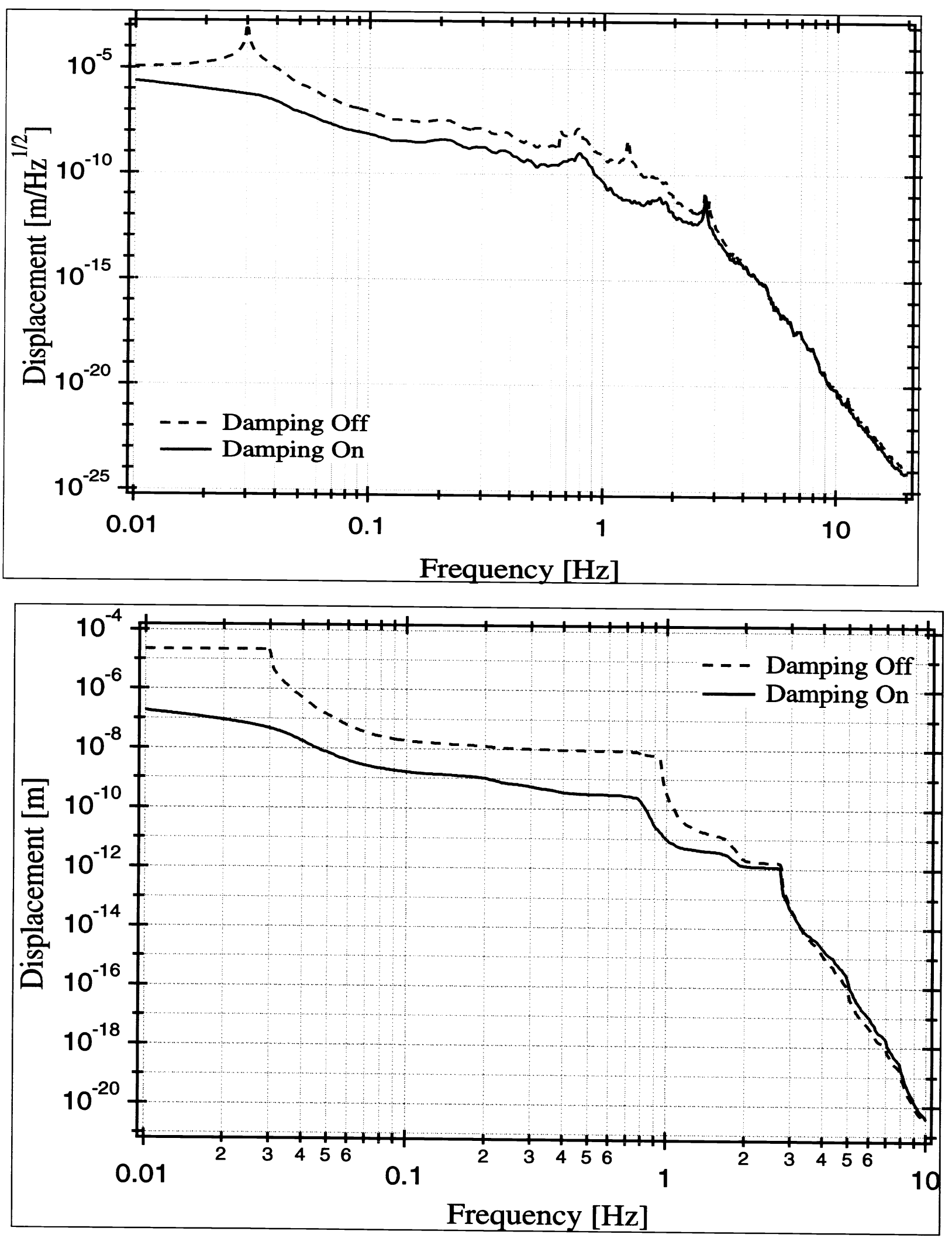

Figure 5: Attenuation (top) and integrated (bottom) Residual motion expected performance of the TAMA SAS towers. 
In GASFs separate blades are joined to the central load disk by flex joint wires thus allowing easy exchange of blade number and strength to match any payload. In MGASFs the crown of blades is monolithic and any change of the payload requires the use of a new, tailored, spring crown. Instead of fine tuning the spring $s$ lift the payload is tuned with small ballast masses. Despite their somewhat lower flexibility of use the MGASFs present several important advantages are:

- Better attenuation factor

- Immunity from some internal resonance effects

- Reduced number of components eliminating potential creak noise sources.

The first two points are evident in figure 4 . The third will be discussed later, the smaller number of components results in simpler filters.

\section{THE MIRROR MULTIPLE PENDULUM}

The bottom of the seismic attenuation chain supports the mirror multiple pendulum in which the suspension material switches from metallic to quartz for thermal noise reasons. The multiple pendulum must provide the necessary control authority to position and aim the mirrors. In order not to inject control noise in the system the actuators on the mirror must themselves be quiet and must have a very small authority. All actuators transfer some of their seismic excitation to the test mass. To operate from a quiet enough actuation platform the actuators are mounted on recoil masses suspended in parallel from the Seismic Attenuation System. The authority allowable (maybe a million times the interferometer dynamic range during lock acquisition but much less during operation) is in no case sufficient to match the off band residual r.m.s. motion of the mirror. Sufficient authority is obtained by hierarchically distributing it over two or three pendula layers. The better the Seismic Attenuation residual motion performance, the less control authority is necessary. Depending on the applications, the SAS performances require only two or three level of control reallocation. For thermal noise reasons, at least a double pendulum suspension is needed anyhow. Thermal noise issues are largely outside the scope of this report.

\section{EXPECTED PERFORMANCE}

All the main components of the passive SAS system have been thoroughly characterized. Simulations of these components have been made, validated and cross -checked with the measured characterization performances.

MSE (Mechanical Simulation Engine) an innovative, modular and comprehensive simulation program ${ }^{10}$ built up with the prevalidated elements has been used to design and estimate the expected performance of attenuation chains. Thanks to the prototype validation of the individual MSE elements, the simulation program is considered particularly reliable. Figure 5 show the expected performance, both in attenuation and in residual motion, of one of the TAMA towers. Prototype tests are ongoing and the TAMA towers will be used in a small test interferometer in the fall 2000 . ConditionalFs to their successful performance they should be installed in TAMA itself in 2001. Similar but larger towers satisfying the LIGO II requirements with a comfortable margin have been designed and prototyped.

\section{EXCESS NOISE CONTROL}

Several factors that may introduce excess noise in the system have been identified and must be eliminated or controlled in a good SI system. Excess mechanical or electromechanical noise may produce events well outside the Gaussian noise distribution of the interferometer and impair its capability to detect transient events. Some of these factors are mechanical noise from the SI chain itself, from cabling, electrical and electromechanical noise on the SI active components and on the mirror actuators, ${ }^{11}$. Some of the solutions are listed below.

The main known sources of mechanical noise are creep and creak, both introducing noise in the entire audio frequency range. Creep noise is generated by localized yield in grains of stressed materials in the structure: it induces detectable acoustic emission and droop. Creep is initiated by dislocations in metal grains that, pushed by the stress field, accumulate on the grain surfaces until the local stress is released by a sudden shift. It was measured ${ }^{12}{ }^{13}$ that the creep problem can be eliminated from the SI structure by controlling the stress levels in the structure, by using creep free materials in the components under stress $^{14}$, and by annealing the SI chain under load. To avoid creep precipitation hardened alloys are used. In these alloys (Maraging steels, Copper-Beryllium alloys, etc.) the dislocations are chemically frozen in the grain volume by intermetallic precipitations. After the few residual dislocations are piled up against the precipitations by an annealing process under stress the acoustic emission stops.

Creak noise is produced when shear stress is applied between two contact surfaces. Most creak noise is avoided by careful design of all contact surfaces perpendicular to the stress direction (see for example the geometry of the link wire at the tips of 
the GASF blades). In most sensitive areas the contact surfaces can be coated with brazing materials to physically connect the contact surfaces. Annealing is expected to eliminate all residual creak noise in well-designed connections.

The most insidious sources of excess noise are connected with the electrical controls. Any active circuit in the SI chain and especially feedback systems may produce mirror noise. Sensors and amplifiers are usually characterized by their r.m.s. noise but have larger spurious non-Gaussian peaks and glitches (due to, for example, cosmic rays crossing gates, shifting charges on the transistor isolators, cable tribo-electricity noise, .). Since the feedback of the circuit operates by nulling its own signal all excess noise is eliminated by counter-shaking the physical system. To avoid this problem:

- All active feedback control systems have been relegated at the beginning of the attenuation chain and isolated from the mirror in the frequency band of interest by the passive isolation chain.

- The authority of any actuator working on or near the mirror is minimized and limited in frequency band by taking advantage of the small mirror residual motion generated by the high-sensitivity, low-frequency accelerometers in the preisolator.

Cables are necessarily plastic components and will generate mechanical noise. Their ill effects are mitigated by their relative softness compared to the rest of the chain structure, but their effect can be substantial at the bottom, in the mirror s multiple pendulum.

Cables also can generate electromechanical noise directly on the mirror actuators by means of piezo- and tribo-electricity and by electronics noise pickup. The best solution is to eliminate them and replace them with wireless controls; substantial work is ongoing on this direction.

\section{CONCLUSIONS.}

Passively attenuating seismic isolation chains fully satisfy the requirements of GWID. These chains were pioneered by the VIRGO collaboration. A second generation of passive SI was developed by LIGO and appears to be the best SI solution for the TAMA and LIGO II interferometers.

Several prototypes of this second generation attenuation structures have been built and are being tested. Their first utilization in an actual interferometer is foreseen in a TAMA test facility in the fall 2000 and in TAMA itself in the following year.

\section{ACKNOWLEDGEMENTS.}

This grant is supported in part by NSF Grant \#PHY9801158.

The results presented in this paper have been generated by the following collaborators: Alessandro Bertolini, Giancarlo Cella, Szabolcs Marka, Flavio Nocera, Kenji Numata, Virginio Sannibale, Akiteru Takamori, Hareem Tariq, Nicolas Viboud, Tatsuo Yoda, Chenyang Wang. Special thanks to our Virgo and TAMA colleagues that continuously supported our work with their ideas and suggestions.

1. http://www.ligo.caltech.edu/

\section{REFERENCES.}

http://www.virgo.infn.it/

http://tamago.mtk.nao.ac.jp/

http://www.anu.edu.au/Physics/ACIGA/

http://www.geo600.uni-hannover.de/

2. R. DeSalvo The quest for gravitational waves, CERN Courier, March 1999. www.cerncourier.com.

3. http://lisa.jpl.nasa.gov/

4. G.Losurdo, Inertial control of the VIRGO superattenuator , to be published in Proc. of the Third E.Amaldi Conference on Gravitational Waves Experiments, CALTECH, Pasadena, 12-16 July 1999. Also gr-qc/9911044. G.Losurdo, Active controls of the VIRGO superattenuator, to be published in in Proc. of the international summer school on experimental physics of gravitational waves, Urbino, 6-18 September 1999. Also gr-qc/0002006

5. G.Losurdo, J.Winterflood, Performance of the Inverted Pendulum as a Preisolator for VIRGO Superattenuator, VIRTRE-PIS-4600-142, 1998. G.Losurdo, et al.: An inverted pendulum pre-isolator stage for VIRGO suspension system , Rev. Sci. Instrum., 70, 2507-2515 (1999).

6. R.De Salvo, et al.: Performances of an ultralow frequency vertical pre-isolator for the VIRGO seismic attenuation chains, Nucl.Instr.and Meth. in Phys.Res. A, 420, 316-335 (1999).

7. M.Beccaria, et al.: Extending the VIRGO gravitational wave detection band down to a few $\mathrm{Hz}$ : metal blade springs and magnetic antisprings, Nucl.Instr.and Meth. in Phys.Res. A, 394, 397-408, 1997. 
8. D.G. Blair et al., Phys. Lett. A 193 (1964), 223. D.G. Blair et al., Rev, Scient. Instr.. 64 (7) (1993), 1899. J. Winterflood et al., Phys. Lett. A 243 (1998), 1.

9. Bertolini, et al., Seismic Noise Filters, Vertical Resonance Frequency Reduction with Geometrical Anti-Springs, a Feasability Study, Nucl. instrum. methods phys. res. A 435 (1999) 475-483.

10. LIGO technical notes: T990106, T990107 and T990108.

11. R. DeSalvo, Non stochastic noise in gravitational wave detectors. Second E. Amaldi conference on Gravitational waves, CERN Geneva 1-4 July 1997, Gravitational Waves, page 228-239, World Scientific, London 1998.

12. Vincenzo Rubino, Studio del Microcreep nelle sospensioni degli Specci di Virgo, Thesis 1996/1997, Dipartimento di Fisica, Universita' di Pisa, Via FilippoBuonarroti 2 Edificio B, I-56127 PISA.

13. G.Cagnoli, at al.: Mechanical shot noise induced by creep in suspension devices, Phys. Lett. A, 237, $21-27$ (1997).

14. M.Beccaria, et al.: The creep problem in the VIRGO suspensions: a possible solution using Maraging steel , Nucl.Instr.and Meth. in Phys.Res. A, 404, 455-469 (1998). 